\title{
DESCARTES' ARGUMENT FÜR \\ DEN PSYCHO-PHYSISCHEN DUALISMUS IM LICHTE DER MODAL-EPISTEMISCHEN LOGIK
}

\author{
Uwe MEIXNER \\ Universität Regensburg
}

In der 6. Meditation (De rerum materialium existentia, et reali mentis a corpore distinctione) der Meditationes de Prima Philosophia formuliert Descartes das folgende Argument:

Et primo, quoniam scio omnia quae clare et distincte intelligo, talia a Deo fieri posse qualia illa intelligo, satis est quod possim unam rem absque altera clare et distincte intelligere, ut certus sim unam ab altera esse diversam, quia potest saltem a Deo seorsim poni; et non refert a qua potentia id fiat, ut diversa existimetur; ac proinde, ex hoc ipso quod sciam me existere, quodque interim nihil plane aliud ad naturam sive essentiam meam pertinere animadvertam, praeter hoc solum quod sim res cogitans, recte concludo meam essentiam in hoc uno consistere, quod sim res cogitans. Et quamvis fortasse (vel potius, ut postmodum dicam, pro certo) habeam corpus, quod mihi valde arcte conjunctum est, quia tamen ex una parte claram et distinctam habeo ideam mei ipsius, quatenus sum tantum res cogitans, non extensa, et ex alia parte distinctam ideam corporis, quatenus est tantum res extensa, non cogitans, certum est me a corpore meo revera esse distinctum, et absque illo posse existere.

(René Descartes, Euvres, hrsg. von Ch. Adam und P. Tannery, Bd. VII, S. 78; im folgenden kurz MP.)

In der Übersetzung von $A$. Buchenau:

Und da ich ja erstlich weiß, daß alles, was ich klar und deutlich denke, in der Weise von Gott geschaffen werden kann, wie ich es denke, so genügt es für mich, ein Ding ohne ein anderes klar und deutlich denken zu können, um mir die Gewißheit zu geben, da $B$ das eine vom anderen verschieden ist, da wenigstens Gott es getrennt setzen kann. Auch kommt es nicht darauf an, durch welche Macht dies geschieht, damit man sie für verschieden hält. Daraus also, da $B$ ich wei $B$, ich existiere, und $\mathrm{da} B$ ich inzwischen bemerke, $\mathrm{da} B$ durchaus nichts anderes zu meiner Natur oder Wesenheit gehöre, als allein, daB ich ein denkendes Ding bin, schließe ich mit Recht, daß meine 
Wesenheit allein darin besteht, daß ich ein denkendes Ding bin. Und wenngleich ich vielleicht - oder vielmehr gewiß, wie ich später auseinandersetzen werde - einen Körper habe, der mit mir sehr eng verbunden ist, so ist doch - da ich ja einerseits eine klare und deutliche Idee meiner selbst habe, sofern ich nur ein denkendes, nicht ein ausgedehntes Ding bin, und andrerseits eine deutliche Idee vom Körper, sofern er nur ein ausgedehntes, nicht denkendes Ding ist - soviel gewiß, $\mathrm{da} B$ ich von meinem Körper wahrhaft verschieden bin und ohne ihn existieren kann.

Dieses Argument soll im folgenden einer Analyse unterzogen werden. Der erste Schritt der Analyse besteht darin, Inhalt und Form des Arguments klar und deutlich herauszuarbeiten, d.h. es zu präzisieren; der zweite Schritt darin, ein Urteil über seine Gültigkeit zu finden. Zu dieser Urteilsfindung sind drei Fragen zu beantworten:

1. die logische Frage: Folgt die Konklusion (das, was bewiesen werden soll) aus den Prämissen (dem, was vorausgesetzt wird)?

2. die inhaltliche Frage: Sind die Prämissen wahr?

3. die pragmatische Frage: Können die Prämissen leichter eingesehen werden als die Konklusion?

Können alle drei Fragen mit "Ja" beantwortet werden, dann ist das Argument gültig; muß dagegen eine mit "Nein" beantwortet werden, so ist es nicht gültig. ${ }^{1}$

Die wichtigste Frage bei der inhaltlichen und formalen Klärung des Arguments ist: Was soll in ihm bewiesen werden? Die Antwort auf diese Frage ist nun keineswegs klar, denn Descartes gibt zwei (dem Wortlaut nach) verschiedene Konklusionen an:

$\mathrm{K} 1$ recte concludo meam essentiam in hoc uno consistere, quod sim res cogitans

$\mathrm{K} 2$ certum est me a corpore meo revera esse distinctum, et absque illo posse existere

Handelt es sich hier also gar nicht um ein Argument, sondern um zwei? - In der Tat formuliert Descartes zu den zwei Konklusionen auch zwei ihnen jeweils entsprechende Prämissen:

P1 ex hoc ipso quod sciam me existere, quodque interim nihil plane aliud ad

1. Im Falle einer bloß pragmatischen Ungültigkeit eines Arguments spricht man besser von seiner Redundanz. 
naturam sive essentiam meam pertinere animadvertam, praeter hoc solum quod sim res cogitans

P2 quia tamen ex una parte claram et distinctam habeo ideam mei ipsius, quatenus sum tantum res cogitans, non extensa, et ex alia parte distinctam ideam corporis, quatenus est tantum res extensa, non cogitans

Weder zu P1 $\rightarrow \mathrm{K} 1$ noch zu $\mathrm{P} 2 \rightarrow \mathrm{K} 2$ paßt hinwiederum dem Wortlaut nach das Schlußprinzip, das Descartes zu Anfang aufstellt:

S Et primo, quoniam scio omnia quae clare et distincte intelligo, talia a Deo fieri posse qualia illa intelligo, satis est quod possim unam rem absque altera clare et distincte intelligere, ut certum sim unam ab altera esse diversam, quia potest saltem a Deo seorsim poni

Angesicht dieser Lage sei die folgende Strategie gefaßt: Es sollen P1 $\rightarrow$ K1, P2 $\rightarrow$ K2 und S je für sich präzisiert werden. Die Präzisierungen von $\mathrm{S}, \mathrm{P} 1 \rightarrow \mathrm{K} 1$ und $\mathrm{P} 2 \rightarrow \mathrm{K} 2$ sollen jedoch logisch möglichst eng miteinander verflochten sein und damit die enge Verflechtung wiederspiegeln, in der Descartes sie selbst formuliert. Allgemein gelte: Eine Präzisierung soll erstens im Ausdeutbarkeitsspielraum des gegebenen Wortlauts bleiben, zweitens mit den schon bekannten Intentionen und

2. A. Beckermann verbindet in seinem Buch Descartes' metaphysischer Beweis für den Dualismus, Freiburg/München 1986, auf S. 68 S mit P2 $\rightarrow$ K2 durch das nach ihm von Descartes stillschweigend vorausgesetzte (weil für diesen selbstverständliche) Prinzip "Wenn ich eine klare und deutliche Idee von a habe, insofern a nur die Eigenschaft $F$, aber nicht die Eigenschaft $G$ hat, und eine (klare und) deutliche Idee von $b$, insofern $b$ nur die Eigenschaft $G$, aber nicht die Eigenschaft $F$ hat, dann begreife ich a klar und deutlich ohne $b$ und b (klar und) deutlich ohne a."

Dabei bedeutet "Ich habe eine klare und deutlich Idee von a, insofern a nur die Eigenschaft F, aber nicht die Eigenschaft $G$ hat" nach Beckermann (ebd., S. 85) für Descartes soviel wie "Ich begreife, da a allein mit der Eigenschaft $F$ und auch ohne die Eigenschaft G existieren kann". - Das angeführte Prinzip ist bei der angeführten Deutung falsch: Sei F die Summe der Eigenschaften der existenten Kugel a. F ist selbst eine Eigenschaft, und sie beeinhaltet die Eigenschaft, eine Kugel zu sein. G dagegen sei die Summe der Eigenschaften der Oberfläche von a. Auch $\mathrm{G}$ ist eine Eigenschaft, und sie beeinhaltet die Eigenschaft, eine Kugeloberfläche zu sein. Ich begreife (klar und deutlich), $\mathrm{da} B$ a allein mit der Eigenschaft $F$ und auch ohne die Eigenschaft $G$ existieren kann, und ich begreife, daB die Oberfläche von a allein mit der Eigenschaft $G$ und auch ohne die Eigenschaft $F$ existieren kann. Aber ich begreife nicht klar und deutlich a ohne die Oberfläche von a. 
Ansichten Descartes' übereinstimmen (d.h. mindestens ihnen nicht widersprechen), drittens ihm gegenüber weitmöglichst fair sein, d.h. seinem Argument die größtmögliche Chance geben, korrekt zu sein. (Diese Richtlinien sind oft nicht gleichzeitig vollständig erfüllbar.) Folgende Abkürzungen werden verwendet:

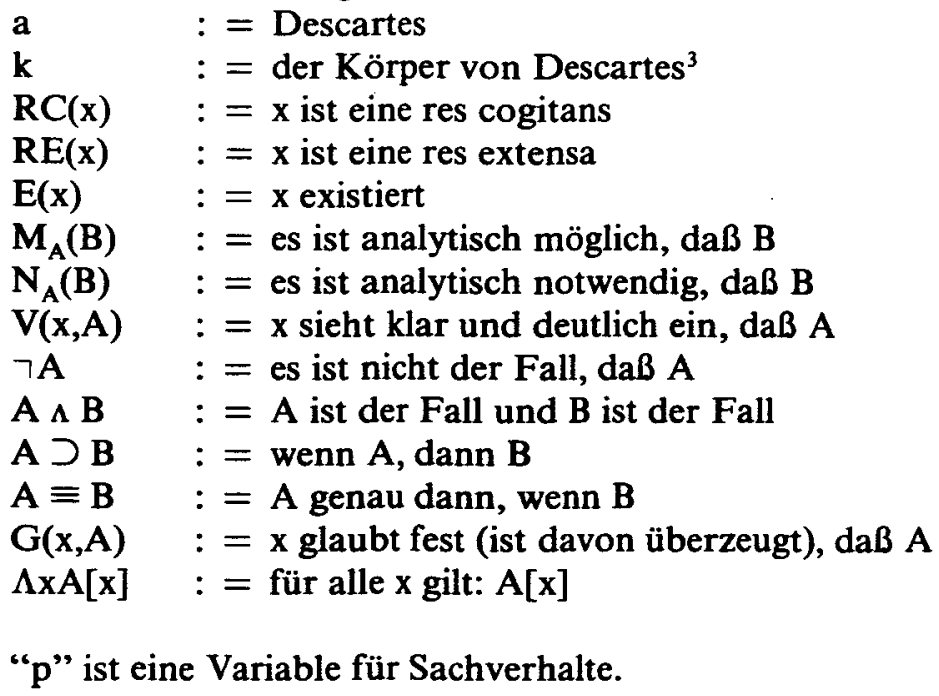

Der textlichen Reihenfolge nach steht nun die Präzisierung von $\mathrm{S}$ an. - A. Buchenau übersetzt - interpretierend - mit Recht "clare et distincte intelligo" in S mit "ich denke klar und deutlich", nicht mit "ich sehe klar und deutlich ein". Descartes will nicht sagen, daß das, was er (klar und deutlich) einsehe, in der Weise von Gott geschaffen werden kann, wie er es einsehe; sondern daß das, was er (klar und

3. " $a$ " und " $k$ " sind hierbei sogenannte Standardnamen (in Kripkes Terminologie: "rigid designators"), d.h. sie bezeichnen in allen möglichen Welten dasselbe Objekt. Modale Aussagen wie " $b$ ist notwendigerweise ein F", "b ist möglicherweise ein G" lassen sich nur, wenn " $b$ " ein Standardname ist, eindeutig als Aussagen über ein Objekt auffassen. - Hier geht es um die Objekte Descartes und seinen Körper, ob diese Objekte gewisse Eigenschaften notwendig haben oder nicht. - Descartes spricht zwar nur von sich und seinem Körper. Damit verbindet sich aber natürlich die stillschweigende Aufforderung an den Leser, seine Argumente bei sich selbst nachzuvollziehen. 
deutlich) als möglich einsehe, in der Weise von Gott (real) geschaffen werden kann, wie er es als möglich einsehe. Etwas (klar und deutlich) als möglich einsehen heißt es (klar und deutlich) denken, nicht etwa es (klar und deutlich) einsehen.

"A kann von Gott geschaffen werden" ist wiederzugeben durch "Es ist analytisch möglich, daß A". ("A" soll also Sachverhalte vertreten; Descartes selbst macht deutlich, daB die Bezugnahme auf Gott nicht wesentlich ist: "et non refert a qua potentia id fiat".) Denn alles, was analytisch möglich ist, kann Gott als allmächtiges, an keine Naturgesetze gebundenes Wesen schaffen; und umgekehrt ist alles, was Gott schaffen kann, wenigstens analytisch möglich. ${ }^{4}$ Es sei davon ausgegangen, daß Descartes durchgängig diesen Möglichkeitsbegriff verwendet.

Die 1. Prämisse des Schlußprinzips $S$ kann also präzisiert notiert werden als:

I $\quad \Lambda p\left(V\left(a, M_{A}(p)\right) \supset M_{A}(p)\right) \quad\left(\right.$ nicht etwa $\Lambda p\left(V(a, p) \supset M_{A}(p)\right)$ !)

Die Probleme beginnen mit der 2. Prämisse von S. - Was heißt es, Dinge $\mathrm{x}$, $\mathrm{y}$ ohne einander klar und deutlich zu denken? Heißt es:

a) klar und deutlich einsehen, daß $\mathrm{x}$ von $\mathrm{y}$ (und damit $\mathrm{y}$ von $\mathrm{x}$ ) räumlich getrennt sein kann?

b) klar und deutlich einsehen, daß x existieren kann, ohne daß y existiert, und daß y existieren kann, ohne daß $\mathrm{x}$ existiert?

c) Klar und deutlich einsehen, daß $x$ existieren kann, ohne daß y ein Teil von $x$ ist, und daß y existieren kann, ohne da $B$ ein Teil von $y$ ist?

Ein Hinweis auf die Präzisierung der 2. Prämisse ergibt sich aus der Konklusion von $\mathrm{S}$, "daß das eine vom anderen verschieden ist". ("da wenigstens Gott es getrennt setzen kann" ist keine zusätzliche Prämisse, sondern eine Zwischenfolgerung aus der 1. und 2. Prämisse, aus der sich - definitorisch äquivalent - die genannte Konklusion

4. Descartes würde die Umkehrung allerdings bestreiten. Nach ihm kann Gott machen, daB ein Widerspruch der Fall ist; ein Widerspruch ist aber analytisch unmöglich. Vgl. hierzu: A. Kenny, The God of the Philosophers, Oxford 1979, S. 18. Mit der Vorstellung einer derartig weitreichenden Allmacht Gottes läBt sich aber kein vernünftiger Sinn mehr verbinden. 
ergibt; siehe unten.) Gemeint ist hier die reale Verschiedenheit, und für diese formuliert Descartes eine Definition:

Duae substantiae realiter distingui dicuntur, cum unaquaeque ex ipsis absque alia potest existere

(MP, S. 162)

In der Übersetzung von A. Buchenau:

Man spricht von der realen Unterschiedenheit zweier Substanzen, wenn jede von ihnen ohne die andere existieren kann

Als Konklusion von S kann man also vorläufig (halb präzisiert) festhalten " $x$ kann ohne $y$ existieren, und $y$ kann ohne $x$ existieren", d.h. “ $M_{A}$ (x existiert ohne y) $\wedge M_{A}$ (y existiert ohne x)". In Anbetracht von I müßte dann die 2. Prämisse halb präzisiert lauten

" $V\left(a, M_{A}\right.$ (x existiert ohne y) $\wedge M_{A}$ (y existiert ohne $\left.x\right)$ )".

(Als unwesentlich vernachlässigt wird hierbei die Tatsache, daB Descartes nicht von "klar und deutlich denken" spricht, sondern nur von "klar und deutlich denken können".)

Insgesamt hat man somit bei der Präzisierung von $\mathrm{S}$ das Zwischenresultat:

I $\quad \Lambda p\left(V\left(a, M_{A}(p)\right) \supset M_{A}(p)\right)$

II $V\left(a, M_{A}\right.$ (x existiert ohne y) $\wedge M_{A}$ (y existiert ohne $\left.x\right)$ )

III $M_{A}$ (x existiert ohne y) $\wedge M_{A}$ (y existiert ohne $x$ )

Schon jetzt sieht man, daß S ein gültiges Schlußprinzip ist, denn es hat die Gestalt

$$
\begin{aligned}
& \Lambda \mathrm{p}\left(\mathrm{V}\left(\mathrm{a}, \mathrm{M}_{\mathrm{A}}(\mathrm{p})\right) \supset \mathrm{M}_{\mathrm{A}}(\mathrm{p})\right) \\
& \mathrm{V}\left(\mathrm{a}, \mathrm{M}_{\mathrm{A}}(\mathrm{B}) \wedge \mathrm{M}_{\mathrm{A}}(\mathrm{C})\right)
\end{aligned}
$$

$M_{A}(B) \wedge M_{A}(C)$

$\mathbf{R}$

$\mathbf{R}$ ist ein $\mathbf{S}$ übergeordnetes gültiges Schlußprinzip, das sich folgendermaßen rechtfertigen läßt:

1. $\Lambda \mathrm{p}\left(\mathrm{V}\left(\mathrm{a}, \mathrm{M}_{\mathrm{A}}(\mathrm{p})\right) \supset \mathrm{M}_{\mathrm{A}}(\mathrm{p})\right) \quad$ Prämisse

2. $V\left(a, M_{A}(B) \wedge M_{A}(C)\right) \quad$ Prämisse 

3. $V\left(a, M_{A}(B)\right) \wedge V\left(a, M_{A}(C)\right)$
aus 2. $\operatorname{mit} \frac{V(a, K \wedge L)}{V(a, K) \wedge V(A, L)}$

4. $V\left(a, M_{A}(B)\right) \supset M_{A}(B)$

5. $V\left(a, M_{A}(C)\right) \supset M_{A}(C)$

Partikularisierung von 1.

6. $M_{A}(B) \wedge M_{A}(C)$

aussagenlogisch aus 3., 4. und 5 .

Was zu klären übrig bleibt, ist der Ausdruck "x existiert ohne y" bzw. "y existiert ohne x". Die Präzisierung im Sinne von b) "x existiert, ohne daß y existiert" bzw. "y existiert, ohne daß x existiert" liegt nahe. Dennoch soll aus gutem Grund dieses Problem noch offen bleiben.

Aus Descartes' Formulierung von $S$ geht hervor, daß er I als wahr ansieht ("Und $d a$ ich ja erstlich weiß, daß alles, was ich klar und deutlich denke ..."); man kann darüberhinaus sagen, daß er es als analytisch wahr ansieht;" das ist für das weitere festzuhalten.

Entgegen der textlichen Reihenfolge sei jetzt das Argument P2 $\rightarrow$ K2 betrachtet. - "claram et distinctam habeo ideam" läßt sich durchaus übersetzen als "ich denke klar und deutlich", d.h. als "ich sehe klar und deutlich als möglich ein". - Was denkt Descartes klar und deutlich, was sieht er klar und deutlich als möglich ein, von was hat er eine klare und deutliche Idee? - (Davon) da $\beta$ er nur ein denkendes Ding ist, kein ausgedehntes, einerseits; (davon) daß der Körper (gemeint ist wohl: sein Körper) nur ein ausgedehntes Ding ist, kein denkendes, andererseits. Demnach kann die Präzisierung von P2 lauten $\left.\left.\left.V\left(a, M_{A}(R C)(a) \Lambda\right\urcorner R E(a)\right) \wedge M_{A}(R E(k) \Lambda\urcorner R C(k)\right)\right)$. Vertretbar ist aber auch $V\left(a, M_{A}(E(a) \wedge R C(a) \wedge\urcorner R E(a)\right) \wedge M_{A}(E(k) \wedge R E(k) \wedge$ $\neg R C(k))$ ); dies paßt besser zu $K 2$, was man präzisieren wird als " $M_{A}(a$ existiert ohne $k$ ) ^ $M_{A}$ (k existiert ohne a)"; denn "a ist von $k$ realiter verschieden" heißt laut Definition soviel wie "a kann ohne $k$ existieren, und k kann ohne a existieren". ${ }^{6}$ Damit also, daß Descartes

5. Freilich verfügt Descartes nicht über den Begriff der Bedeutungswahrheit. Für ihn ist I jedoch ein Prinzip, das er durch bloßes Nachdenken - mit geschlossenen Augen im Lehnstuhl meditierend - einsehen kann.

6. Die Verschiedenheit, die Descartes hier meint, ist also etwas anderes, nämlich stärkeres als die gewöhnliche Verschiedenheit (das kontradiktorische Gegenteil der Identitätsrelation). In der Tat ist " $\mathrm{a} \neq \mathrm{k}$ ", wo " $\neq$ " im selben 
in $\mathrm{K} 2$ sagt, daß er von seinem Körper wahrhaft verschieden ist, ist schon alles gesagt, und die Hinzufügung "und ohne ihn existieren kann" ist logisch gesehen überflüssig. Sie dient nur der Verdeutlichung bzw. Betonung.

Wie läßt sich P2 $\rightarrow$ K2 in der semipräzisierten Form, die es angenommen hat, unter Verwendung des halbpräzisierten $S$ logisch rechtfertigen? - So:

\section{[1. $\left.\Lambda p\left(V\left(a, M_{A}(p)\right) \supset M_{A}(p)\right)\right] \quad$ als analytisch wahr vorausgesetzt ${ }^{7}$}

Sinn verwendet wird wie in " $2 \neq 3$ ", keine Behauptung die, wenn wahr, den Dualismus zur Folge hat, sondern fast eine Trivialität. Darauf weist auch Kripke am Schluß seines Aufsatzes "Identity and Necessity" (in Identity and Individuation, hrsg. von M.K. Munitz, New York 1971, S. 164) hin. Die Frage des Dualismus ist nicht, ob man von seinem Körper verschieden (im gewöhnlichen Sinn von "verschieden") ist, sondern mindestens, ob man ohne seinen Körper existieren kann, wenn nicht gar, ob man ohne irgendeinen Körper existieren kann. Insofern geht Kripkes Verteidigung von Descartes' Argument etwas an der Sache vorbei, denn eine derartig schwache These wie, daß $a \neq k$, wollte Descartes gar nicht zeigen, sondern vielmehr (u. a.) die These, die Kripke als Descartes' Prämisse anführt, nämlich daß er ohne seinen Körper existieren kann. Wer freilich dies als Prämisse akzeptiert, muß auch $a \neq k$ akzeptieren (bei Deutung von " $a$ " und " $k$ " als starre Designatoren), wie Kripke gezeigt hat. - M. Gueroult schreibt in Descartes selon l'ordre des raisons, II, Paris 1968, auf S. 67: "Ce fait que la preuve de la distinction [von Körper und Seele] ne peut s'accomplir qu'à l'encontre du témoignage du sens, nous permet d'entrevoir qu'elle aura pour conséquence de limiter les prétensions de ce dernier." Descartes will aber mit seinem Beweis, anders als Parmenides, nichts beweisen, was den Sinnen widerspricht. Daß Körper und Seele miteinander verbunden sind, ist ein Faktum, was auch für Descartes durch die Erfahrung, nämlich die der psycho-physischen Wechselwirkung, feststand. Ihm geht es vielmehr um die Frage, ob sie auch ohne einander existieren können, d.h. nach Descartes' Definition real verschieden sind. Dies ist das, was er beweisen will, und dies steht in keinem Widerspruch zum Zeugnis der Sinne. Daher hat es Descartes auch nicht nötig, sich in seinem Beweis auf die Allmacht Gottes zu berufen, wie Gueroult meint (ebd. S. 64); die Rede von der Allmacht Gottes soll, wie gesagt, nur zum Ausdruck bringen, daß er den schwächst möglichen Begriff des Möglichseins verwendet.

7. Deshalb von Descartes weggelassen, was durch die Einklammerung angedeutet werden soll. 
2. $\left.\left.V\left(a, M_{A}(E(a) \wedge R C(a) \wedge\urcorner R E(a)\right) \wedge M_{A}(E(k) \wedge R E(k) \wedge\urcorner R C(k)\right)\right) P 2$

3. $V\left(a, M_{A}\right.$ (a existiert ohne $\left.k\right) \wedge M_{A}$ (k existiert ohne a)) aus 2 .

4. $\mathrm{M}_{\mathrm{A}}$ (a existiert ohne $\left.\mathrm{k}\right) \wedge \mathrm{M}_{\mathrm{A}}$ (k existiert ohne a) aus $1 ., 3$. mit $\mathrm{S}$

Der Schritt von 2. zu 3., den Descartes doch machen muß, um S anwenden zu können, gibt nun einen Hinweis darauf, was unter "a existiert ohne k" und " $k$ existiert ohne a" zu verstehen ist; denn dieser Schritt ist legitim, wenn "a existiert ohne k" aus "a existiert als res cogitans, ohne eine res extensa zu sein" analytisch folgt, und ebenso "k existiert ohne a" aus "k existiert als res extensa, ohne eine res cogitans zu sein". Dies gibt Anlaß zu den folgenden Definitionen, die " $x$ existiert ohne y", $\mathrm{RE}(\mathrm{x}), \mathrm{RC}(\mathrm{x})$ in solcher Weise erklären, daß der Schritt von 2. zu 3. mit der erwähnten Begründung legitim ist:

$x$ existiert mit $y \quad:=E(x) \wedge y$ ist mit $x$ korreliert ("verbunden") $\wedge E(y)$

$x$ existiert ohne $y:=E(x) \wedge(\neg(y$ ist mit $x$ korreliert $) v \neg E(y))$

$\mathrm{RE}(\mathrm{x}) \quad:=\mathrm{Vy}(\mathrm{y} \text { ist ein Körper } \Lambda \mathrm{y} \text { ist mit } \mathrm{x} \text { korreliert })^{8}$

$\mathrm{RC}(\mathrm{x}) \quad:=\mathrm{Vy}(\mathrm{y}$ ist eine Person $\mathrm{\Lambda} \mathrm{y}$ ist mit $\mathrm{x}$ korreliert $)$

Die Natur der Korrelationsrelation sei hier weitgehend offengelassen; es soll aber analytisch gelten:

$\Lambda \mathrm{x} \Lambda \mathrm{y}(\mathrm{x}=\mathrm{y} \supset \mathrm{x}$ ist mit $\mathrm{y}$ korreliert $)$

Gemäß dieser Definitionen gilt, wegen $a$ ist eine Person und $k$ ist ein Körper:

B1

E(a) $\Lambda \neg R E(a) \supset$ a existiert ohne $k$

B2

E(k) $\wedge \neg R C(k) \supset$ k existiert ohne a

8. "V" ist hier der Existenzoperator. 
Beweis:

i) ang. $E(a) \wedge\urcorner R E(a)$, also:

x) $E(a)$;

$\mathbf{x x}) \neg(\mathbf{k}$ ist mit a korreliert), denn wegen $\neg \mathrm{RE}$ (a) laut Definition $\Lambda \mathrm{y}(\mathrm{y}$ ist ein Körper $\supset \neg(\mathrm{y}$ ist mit a korreliert $))$; nun $k$ ist ein Körper; also $\neg$ ( $(\mathrm{k}$ ist mit a korreliert); aus $\mathrm{x}$ ) und $\mathrm{xx}$ ) folgt laut Definition $a$ existiert ohne $k$;

ii) entsprechend.

Aufgrund dieser Definitionen und dem erwähnten Prinzip gilt außerdem analytisch:

$\Lambda \mathrm{x}(\mathrm{x}$ ist eine Person $\supset \mathrm{RC}(\mathrm{x})) \quad \Lambda \mathrm{x}(\mathrm{x}$ ist ein Körper $\supset \mathrm{RE}(\mathrm{x}))$

Analytisch sind die Beziehungen B1 und B2 freilich nur, wenn $a$ ist eine Person und $k$ ist ein Körper analytisch notwendige Sachverhalte sind, wenn eine Person zu sein eine essentielle Eigenschaft von a ist und ein Körper zu sein eine essentielle Eigenschaft von k. - Man kann davon ausgehen, daß Descartes dies annimmt; es sei hier auch als richtig anerkannt. ${ }^{9}$

Wie steht es schließlich mit dem Argument P1 $\rightarrow$ K1? - Meint Descartes in K1 tatsächlich, was er wörtlich sagt: daß seine einzige essentielle Eigenschaft die ist, ein denkendes Ding zu sein? Ist es nicht etwa auch eine essentielle Eigenschaft von ihm, daß er mit sich selbst identisch ist, und sollte er das übersehen haben? Worauf es ihm sicherlich allein ankommt, ist, daß es eine essentielle Eigenschaft von ihm ist, eine res cogitans zu sein, aber keine, eine res extensa zu sein, d.h. $N_{A}(R C(a)) \wedge \neg N_{A}(\operatorname{RE}(a)) .^{10}$

9. Falls das Wort "Person" bei Descartes nicht vorkommt, so bedeutet das nicht, daB er den Personbegriff nicht verwendet. Er kann ja einen anderen Namen für ihn haben. (So verhält es sich in der Tat.) Der Personbegriff Descartes' kann zudem ein weitgehend anderer sein als der unsrige. (Auch das ist tatsächlich gegeben.) Zum Existieren als res cogitans und also zum Existieren als Person gehört aber für ihn wie für uns zu zweifeln, zu bejahen, zu verneinen, zu wollen etc. (vgl. MP, S. 28, 2. Meditation, und S. 34, Anfang der 3. Meditation).

10. Zu Descartes' Verständnis von Essenz und essentieller Eigenschaft 
Präzisiert man $\mathrm{K} 1$ in dieser Weise, so wird man P1 als $V\left(a, N_{A}(R C(a)) \wedge \neg N_{A}(R E(a))\right)$ wiedergeben; " animadvertam" dürfte dort nur - der Variation halber - "clare et distincte intelligam" vertreten, und die Existenz Descartes' fügt $V\left(a, N_{A}(R C(a)) \wedge \neg N_{A}\right.$ (RE(a))) nichts hinzu, sondern folgt vielmehr analytisch daraus; ${ }^{11}$ durch die Erwähnung seiner Existenz will Descartes nur daran erinnern und betonen, daß diese die notwendige Bedingung dafür ist, $\operatorname{daß} V\left(a, N_{A}(R C(a)) \wedge \neg N_{A}(R E(a))\right)$.

Unter Verwendung des $\mathrm{S}$ übergeordneten Schlußprinzips $\mathrm{R}$ ließe sich dann P1 $\rightarrow$ K1 logisch rechtfertigen:

[1. $\left.\Lambda p\left(V\left(a, M_{A}(p)\right) \supset M_{A}(p)\right)\right]$

2. $V\left(a, N_{A}(R C(a)) \wedge \neg N_{A}(R E(a))\right)$

3. $V\left(a, M_{A}\left(N_{A}(R C(a))\right) \wedge M_{A}\left(\neg N_{A}(R E(a))\right)\right)$

4. $M_{A}\left(N_{A}(R C(a))\right) \wedge M_{A}\left(\neg N_{A}(R E(a))\right)$

5. $N_{A}(R C(a)) \wedge \neg N_{A}(R E(a))$ als analytisch wahr

vorausgesetzt

P1

modal-epistemisch aus 2.

aus 1. und 3. mit $R$

modallogisch aus 4 .

Diese Deduktion ist freilich schwerlich Descartes' eigene, denn das setzte voraus, daß ihm die modallogischen Gesetze

vergl. Lilli Alanen, Studies in Cartesian Epistemology and Philosophy of Mind, Helsinki 1982, S.26f. Sie schreibt dort: "Essence (essentia), as opposed to accident (accidens), designates the necessary and permanent properties of a thing ... Descartes seems to be conforming to this traditional usage". Wenn dem so ist, so kann Descartes nicht wohlbedacht davon sprechen, daß seine Essenz in der einzigen Eigenschaft, zu denken, besteht, denn diese Eigenschaft hat von ihr verschiedene Teileigenschaften, die Descartes mit ihr ebenfalls notwendigerweise hat und die daher auch zu seiner Essenz gehören. Allerdings könnte er meinen, daß seine Essenz durch die Eigenschaft des Denkens erschöpft wird, in dem Sinne, daß alle seine essentiellen Eigenschaften in ihr enthalten sind. Wie dem auch sei, für das, was Descartes zeigen will (die reale Verschiedenheit von Körper und Person), benötigt er nicht die problematische Annahme, daB sein Wesen einzig im Denken besteht.

11. "Nam, si judico ceram existere, ex eo quod hanc videam, certe multo evidentius efficitur me ipsum etiam existere, ex eo ipso quod hanc videam" (MP, S. 33, 2. Meditation) 


$$
\begin{gathered}
94 \\
M_{A}\left(N_{A}(B)\right) \supset N_{A}(B) \text { und } M_{A}\left(\neg N_{A}(B)\right) \supset \neg N_{A}(B)
\end{gathered}
$$

wenigstens implizit) bekannt sind, was etwas viel verlangt ist. (Seine Vertrautheit mit $\mathbf{R}$ ist dagegen unproblematisch.)

Nimmt man als cartesische Schlußprinzipien

$$
\begin{aligned}
& \text { R1 } \Lambda p\left(V\left(a, M_{A}(p)\right) \supset M_{A}(p)\right) \\
& \mathrm{V}\left(\mathrm{a}, \mathrm{M}_{\mathrm{A}}(\mathrm{B})\right) \\
& M_{A} \text { (B) } \\
& \text { R2 } \Lambda \mathrm{p}\left(\mathrm{V}\left(\mathrm{a}, \mathrm{N}_{\mathrm{A}}(\mathrm{p})\right) \supset \mathrm{N}_{\mathrm{A}}(\mathrm{p})\right) \\
& \mathrm{V}\left(\mathrm{a}, \mathrm{N}_{\mathrm{A}}(\mathrm{B})\right) \\
& \mathrm{N}_{\mathrm{A}} \text { (B) }
\end{aligned}
$$

an, so sieht die logische Rechtfertigung von $\mathrm{P} 1 \rightarrow \mathrm{K} 1$ so aus:

[1. $\left.\left.\Lambda \mathrm{p}\left(\mathrm{Va}, \mathrm{M}_{\mathrm{A}}(\mathrm{p})\right) \supset \mathrm{M}_{\mathrm{A}}(\mathrm{p})\right)\right] \quad$ als analytisch wahr vorausgesetzt

[2. $\left.\Lambda \mathrm{p}\left(\mathrm{V}\left(\mathrm{a}, \mathrm{N}_{\mathrm{A}}(\mathrm{p})\right) \supset \mathrm{N}_{\mathrm{A}}(\mathrm{p})\right)\right] \quad$ stillschweigend als analytisch wahr vorausgesetzt

3. $V\left(a, N_{A}(R C(a)) \wedge \neg N_{A}(R E(a))\right) P 1$

4. $\mathrm{V}\left(\mathrm{a}, \mathrm{N}_{\mathrm{A}}(\mathrm{RC}(\mathrm{a}))\right)$

5. $\mathrm{V}\left(\mathrm{a}, \mathrm{M}_{\mathrm{A}}(\neg \mathrm{RE}(\mathrm{a}))\right)$

6. $\mathrm{N}_{\mathrm{A}}(\mathrm{RC}(\mathrm{a}))$

7. $\mathrm{M}_{\mathrm{A}}(\neg \mathrm{RE}(\mathrm{a}))$

8. $N_{A}(\operatorname{RC}(a))_{\Lambda} \neg N_{A}(\operatorname{RE}(a))$ aus 3. mit $\frac{V(a, K \wedge L)}{V(a, K)}$ aus 3. mit $\left.\frac{V(a, K \wedge L)}{V(a, L)}, \neg N_{A}=M_{A}\right\urcorner$ aus 2. und 4. mit R2 aus 1. und 5. mit R1 aus 6. und 7. aussagenlogisch, $\left.\mathbf{M}_{\mathbf{A}}\right\urcorner=\neg \mathbf{N}_{\mathbf{A}}$

R1 und R2 sowie die Überzeugung von der ersten Prämisse von R2 ${ }^{12}$ als analytisch wahr darf man Descartes sicherlich zumuten.

Hier ist zwar vom Sehen statt vom Einsehen die Rede, der Unterschied ist jedoch nicht wesentlich.

12. Die erste Prämisse von R2 ergibt sich logisch aus Descartes' immer 
Es wurde schon festgestellt, daß $R$ und also $S$ ein gültiges Schlußprinzip ist; dasselbe gilt ganz offensichtlich auch von $R 1$ und R2; sowohl $\mathrm{P} 1 \rightarrow \mathrm{K} 1$ als auch $\mathrm{P} 2 \rightarrow \mathrm{K} 2$ sind also logisch korrekt. Ob man nun auch die Voraussetzungen, von denen Descartes in beiden Argumenten ausgeht als wahr ansehen kann, wird entscheidend davon abhängen, welche Deutung man dem Operator V ("sieht klar und deutlich ein") verleiht. Dies ist der Rest an Präzisierungsarbeit, der zu leisten noch übrig ist.

Es wurde bereits angenommen, daß für V folgende Schlußprinzipien gelten:

$\mathrm{V}(\mathrm{x}, \mathrm{B})$

$\underline{N_{A}(B \supset C)}$

$\mathrm{V}(\mathbf{x}, \mathrm{C})$

$V(x, B \wedge C)$

$\mathrm{V}(\mathrm{x}, \mathrm{B}) \wedge \mathrm{V}(\mathrm{x}, \mathrm{C})$

(bzw. $V(x, B)$,

bzw. $V(x, C))$ (in der logischen Rechtfertigung von $\mathrm{P} 2 \rightarrow \mathrm{K} 2^{13}$ und in der ersten logischen Rechtfertigung von $\mathrm{Pl}$ $\rightarrow \mathrm{K} 1$ )

(in der zweiten logischen Rechtfertigung von $\mathrm{P} 1 \rightarrow \mathrm{K} 1$ und in der Rechtfertigung von $\mathrm{R}$ )

Soweit unterscheidet sich $\mathrm{V}$ nicht von $\mathrm{G}$, dem Operator rationalen

wieder reiteriertem Prinzip, daß alles, was man klar und deutlich einsieht, wahr ist. Sie ist aber logisch schwächer als dieses Prinzip und daher auch nicht so problematisch. Man unterscheide sie sorgfältig von dem $\operatorname{Satz} \Lambda \mathrm{p}\left(\mathrm{N}_{\mathrm{A}}\right.$ $\left.(V(a, p)) \supset N_{A}(p)\right)$ - "Was ich einsehen muB, ist notwendigerweise wahr" -, demgegenüber Descartes mit Recht in der fünften Meditation betont, daß unser Denken den Dingen keine Notwendigkeit auferlegt (vergl. MP, S.66). An anderen Stellen macht er aber von diesem Satz durchaus Gebrauch; den Gedankengang des Hauptteils der 2. Meditation könnte man zusammenfassen in der Aussage: "Ich kann nicht daran zweifeln, daß ich ein denkendes Ding bin; also bin ich ein notwendigerweise (essentiell) denkendes Ding". (Vergl. M.D. Wilson, Descartes, London 1978, S.72.)

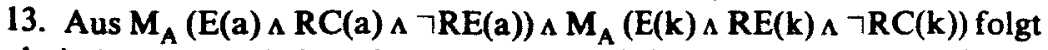
analytisch “ $M_{A}$ (a existiert ohne $k$ ) $\wedge M_{A}$ (k existiert ohne a)", da "a existiert ohne $k$ " analytisch aus $E(a) \wedge \neg R E(a)$ folgt und " $k$ existiert ohne $a$ " aus $E(k) \wedge$ \urcorner $R C(k)$, gemäß der gegebenen Definitionen und $N_{A}$ (a ist eine Person), $N_{A}(k$ ist ein Körper). 
Glaubens (im Sinne von Überzeugtsein). Könnte man definieren $\mathrm{V}(\mathrm{x}, \mathrm{B}):=\mathrm{G}(\mathrm{x}, \mathrm{B}) ?^{14}$

In der Tat gehen bei dieser Deutung $\Lambda p\left(V\left(a, M_{A}(p)\right) \supset M_{A}(p)\right)$ und $\Lambda \mathrm{p}\left(\mathrm{V}\left(\mathrm{a}, \mathrm{N}_{\mathrm{A}}(\mathrm{p})\right) \supset \mathrm{N}_{\mathrm{A}}(\mathrm{p})\right)$ in analytisch wahre Sätze über; dies ergibt sich aus den Bedeutungspostulaten für rationalen Glauben, insbesondere $N_{A}(B) \supset G(x, B)$. - Sind im Lichte dieser Deutung auch $P 1$ und $\mathrm{P} 2$ als wahr anzusehen?

Es besteht kein Grund daran zu zweifeln, daß Descartes faktisch davon überzeugt ist, zum einen, daß

T1

$$
\left.M_{A}(E(a) \wedge R C(a) \wedge \neg R E(a)) \wedge M_{A}(E(k) \wedge R E(k) \wedge\urcorner R C(k)\right),
$$

zum anderen, daß

T2

$$
\mathrm{N}_{\mathrm{A}}(\mathrm{RC}(\mathrm{a})) \wedge \neg \mathrm{N}_{\mathrm{A}}(\mathrm{RE}(\mathrm{a})) \quad(=\mathrm{K} 1)
$$

Aber daraus, da $\beta$ Descartes faktisch davon überzeugt ist, folgt nicht, $\mathrm{da} ß$ er rational davon überzeugt ist. Dazu ist vielmehr neben der inneren Überzeugungshaltung auch notwendig, daß das, wovon er überzeugt ist, analytisch möglich ist. - Andererseits benötigt man Descartes' rationale Überzeugtheit von $T 1$ und $T 2$, denn für $V$ im Sinne von faktischer Überzeugtheit sind die Sätze $\Lambda \mathrm{p}\left(\mathrm{V}\left(\mathrm{a}, \mathrm{M}_{\mathrm{A}}(\mathrm{p})\right) \supset\right.$ $\left.M_{A}(p)\right)^{15}$ und $\Lambda p\left(V\left(a, N_{A}(p)\right) \supset N_{A}(p)\right)$ nicht analytisch wahr und aller Wahrscheinlichkeit nach sogar falsch; auch ein durchaus scharf-

14. Diese Definition ist nicht vollständig in Descartes' Sinne, denn im weiteren Kontext der Meditationes (insbesondere in der 2. Meditation) wird deutlich, daß für ihn nicht nur das Prinzip $\Lambda p\left(V\left(a, M_{A}(p)\right) \supset M_{A}(p)\right) b z w$. $\Lambda \mathrm{p}\left(\mathrm{V}\left(\mathrm{a}, \mathrm{N}_{\mathrm{A}}(\mathrm{p})\right) \supset \mathrm{N}_{\mathrm{A}}(\mathrm{p})\right)$; sondern sogar das stärkere Prinzip $\Lambda \mathrm{p}(\mathrm{V}(\mathrm{a}, \mathrm{p}) \supset \mathrm{p})$ analytisch wahr ist. Klar und deutlich einsehen ist somit offenbar ein wissensähnlicher, kein bloB glaubensähnlicher Begriff. Nun verwendet aber Descartes an der zur Diskussion stehenden Stelle nicht das Prinzip $\Lambda p(V(a, p)$ $\supset \mathrm{p})$, sondern nur das Prinzip $\Lambda \mathrm{p}\left(\mathrm{V}\left(\mathrm{a}, \mathrm{M}_{\mathrm{A}}(\mathrm{p})\right) \supset \mathrm{M}_{\mathrm{A}}(\mathrm{p})\right)$ bzw. $\Lambda \mathrm{p}\left(\mathrm{V}\left(\mathrm{a}, \mathbf{N}_{\mathrm{A}}(\mathrm{p})\right)\right.$ $\supset \mathrm{N}_{\mathrm{A}}(\mathrm{p})$ ); wie sich zudem zeigen wird, ergeben sich für die Einsehbarkeit von $P 1$ und $P 2$ gewisse Schwierigkeiten bei der angegebenen Deutung von $V(x, B)$; der Struktur nach gleiche, aber dem Grade nach größere Schwierigkeiten resultieren bei allen logisch stärkeren Deutungen von $\mathrm{V}(\mathbf{x}, \mathbf{B})$.

15. In seinem Aufsatz "Descartes's Denial of Mind-Body Identity" (in Descartes: Critical and Interpretive Essays, hrsg. von M. Hooker, Baltimore 
sinniger Denker wie Descartes ist in begrifflich-analytischen Fragen nicht unfehlbar. Tatsächlich ist der Weg, auf dem Descartes etwa zu seiner Überzeugung von T2 gelangt, logisch brüchig. Er schließt nämlich aufgrund seiner Zweifelsbetrachtung so:

"Ich kann nicht daran zweifeln, daß ich ein denkendes Ding bin; also bin ich notwendig ein denkendes Ding."

"Ich kann daran zweifeln, daß ich ein ausgedehntes Ding bin; also bin ich möglicherweise kein ausgedehntes Ding."

Die modal-epistemischen Schlußprinzipien, die hier verwendet werden, sind:
$N_{A}(G(a, B))$
$\mathrm{M}_{\mathrm{A}}(\neg \mathrm{G}(\mathrm{a}, \mathrm{B}))$
$\mathbf{N}_{\mathbf{A}}$ (B)
$\mathbf{M}_{\mathrm{A}}(\neg \mathrm{B})$

Nur das zweite ist gültig (bei Voraussetzung eines rationalen Glaubensbegriffs) ${ }^{16}$; das erste ist ungültig. Auch Descartes hätte dies

1978) wendet sich $M$. Hooker auf $S .178$ gegen das Prinzip $\Lambda p\left(V\left(a, M_{A}(p)\right) \supset\right.$ $\left.M_{A}(p)\right)$ und auf S. 176f. gegen das stärkere Prinzip $\Lambda p\left(M_{A}\left(V\left(a, M_{A}(p)\right)\right) \supset\right.$ $M_{A}(p)$ ), bei ihm: (p) (if $p$ is conceivable, $p$ is possible)(siehe ebd. $S$. 176). Seine Argumente sind gegenstandslos, wenn " $p$ is conceivable" nicht im Sinne von "faktisch davon überzeugt sein können, daß p möglich ist" genommen wird, sondern im Sinne von "rational" davon überzeugt sein können, daß p möglich ist"; bzw. wenn "p is being conceived" nicht im Sinne von "faktisch davon überzeugt sein, daß p möglich ist" genommen wird, sondern im Sinne von "rational davon überzeugt sein, daß p möglich ist".

16. Bei Verwendung eines faktischen Glaubensbegriffes ist es ungültig: Es ist analytisch möglich, daran faktisch zu zweifeln (faktisch nicht zu glauben), daB die Winkelsumme im Dreieck $180^{\circ}$ ist; doch es ist nicht analytisch möglich, daß sie nicht $180^{\circ}$ ist. - Genau in diese Richtung geht mit einem ähnlichen Gegenbeispiel ein Einwand Arnaulds (MP, S. 201f.; vgl. hierzu auch F. v. Kutschera, Grundfragen der Erkenntnistheorie, Berlin 1982, S. 297f.). Der Einwand sticht nur, wenn Descartes einen faktischen Glaubensbegriff verwendet, was aber aufgrund der angenommenen Geltung des Prinzips $\Lambda \mathrm{p}\left(\mathrm{V}\left(\mathrm{a}, \mathrm{M}_{\mathrm{A}}(\mathrm{p})\right) \supset \mathrm{M}_{\mathrm{A}}(\mathrm{p})\right)$ bei $V(x, A:=G(x, A)$ sicher nicht der Fall ist.

A. Beckermann schreibt in Descartes' metaphysischer Beweis für den Dualismus auf S. 63, daß nur die Verwechslung von epistemischer und metaphysischer (objektiver) Möglichkeit den Übergang von "Ich kann daran zweifeln, 
auffallen können; denn da es für ihn auch unbezweifelbar ist, daß er existiert, ergäbe sich mit dem ersten Schlußprinzip, daß er — wie Gott - notwendigerweise existiert.

Kann man nicht doch einsehen, ohne daß man $\mathrm{K} 1$ oder $\mathrm{K} 2$ schon als wahr annimmt, daß Descartes von $\mathrm{T} 1$ und $\mathrm{T} 2$ rational überzeugt ist, daß P1 und P2 wahr sind? Dazu bräuchte man nur noch einzusehen, $\mathrm{daB} \mathrm{M}_{\mathrm{A}}$ (T1) und daß $\mathrm{M}_{\mathrm{A}}$ (T2). Wegen der Verschachtelung der Modaloperatoren ist dies nicht leicht und wohl für niemanden leichter als T1 und T2 selbst einzusehen, zumal man in der Modallogik (S5) beweisen kann, daß $M_{A}(T 1) \equiv T 1$ und daß $M_{A}(T 2) \equiv T 2$.

Nun wurde festgestellt, daß Descartes mit analytischer Notwendigkeit eine Person ist und der Körper von Descartes mit analytischer Notwendigkeit ein Körper. Daraus folgt, daß Descartes mit analytischer Notwendigkeit eine res cogitans ist und sein Körper mit analytischer Notwendigkeit eine res extensa. Damit ergibt sich, daß T2 analytisch äquivalent ist mit

T2' $\left.7 \mathrm{~N}_{\mathrm{A}} \mathrm{RE}(\mathrm{a})\right)$

und daß $T 1$ analytisch äquivalent ist mit

$T 1^{\prime} \quad M_{A}(E(a) \wedge \neg R E(a)) \wedge M_{A}(E(k) \wedge \neg R C(k))$.

T2 folgt also aus $T 1$, da $T 2$ ' aus $T 1^{\prime}$ folgt. $T 1$ ' aber ist eine außerordentlich schwache dualistische These, der man kaum die Zustimmung versagen kann. ${ }^{17} \mathrm{Um}$ in der Sprache Descartes' zu reden:

daß ich einen Körper habe" zu "Es ist möglich, daß ich keinen Körper habe" ermögliche, und weist in diesen Zusammenhang auf Arnaulds Einwand hin. Dies ist unrichtig; die Termini des angeführten Schlusses lassen sich sinnvoll in solcher Weise deuten, daß er nicht nur gültig scheint, sondern gültig ist.

17. Descartes selbst betont die Bescheidenheit seines Dualismus. Arnauld meint in seinen Einwänden, da $B$ das dualistische Argument in der 6. Meditation zuviel zu beweisen und nur auf die platonische Meinung hinzuführen scheine, nach der nichts Körperliches zu unserer Wesenheit gehört, so daß der Mensch nur ein Geist ist, der Körper dagegen bloß das Vehikel des Geistes. (MP, S. 203)

Descartes antwortet (in der Übersetzung von A. Buchenau): "Ich sehe auch 
Wenigstens Gott könnte es so einrichten, daß Descartes existiert, ohne da $ß$ ein Körper mit ihm verbunden ist; daß sein Körper existiert, ohne $\mathrm{da} B$ eine Person mit ihm verbunden ist. Wer wollte - prima facie das bestreiten? Nicht einmal die modernen Materialisten, denen die Annahme einer "analytisch notwendigen Identität" von körperlichen und seelischen Zuständen unhaltbar scheint und die es stattdessen für möglich halten, von einer "kontingenten Identität" derselben zu sprechen. (Zur Kritik vgl. F. v. Kutschera, Grundfragen der Erkenntnistheorie, S. 266ff.) Soll freilich $\mathrm{Tl}^{\prime}$ gelten, so darf aus der Existenz von Descartes (d.h. aus der Existenz von Descartes als Person - da er analytisch notwendig eine ist - und also als res cogitans) nichts analytisch folgen, wofür seinerseits es analytisch notwendig ist, daß ein Körper mit Descartes korreliert ist. Das Wort "Person" verwendet man heute aber in dem Sinne, daß wohl mit analytischer Notwendigkeit jede existierende Person sich an einem bestimmten physikalischen Ort befindet, wofür seinerseits analytisch notwendig ist, daß ein Körper mit ihr korreliert ist; wie sollte etwas einen Ort haben, womit kein Körper verbunden ist? ${ }^{18}$

Somit ist klar, daB Descartes $N_{A}$ (a ist eine Person) nicht in

\begin{abstract}
nicht inwiefern dieses Argument 'zu viel beweisen soll', denn es kann nichts weniger gesagt werden, um zu zeigen, daß eine Sache real von der anderen verschieden ist, als daß durch die göttliche Macht eine von der anderen getrennt werden kann. Ich glaube mich aber genügend in acht genommen zu haben, damit niemand daraufhin glauben könnte, 'daß der Mensch nur ein Geist ist, der sich des Körpers bedient'." (MP, S. 227f.)

Descartes selbst hätte das "Dogma vom Gespenst in der Maschine" abgelehnt, das ihm von G. Ryle in The Concept of Mind, London 1949, in grober Vereinfachung und unter Ignorierung (Ignoranz?) seiner Argumente unterstellt wird.

18. Damit dies gilt, tut man gut daran, den Körperbegriff nicht zu eng zu fassen. Auch Schatten, Lichtfelder, Astralleiber etc. sollten Körper sein. Ja, man sieht sich gezwungen, den Körperbegriff ganz im Sinne Descartes' aufzufassen, nach dem alle ausgedehnten Entitäten, also auch immaterielle geometrische Figuren, Körper sind. Die Materialität existierender Personen (im modernen Sinn) wird bei näherem Zusehen durch das genannte analytische Merkmal existierender Personen (im modernen Sinn) keineswegs für diese analytisch notwendig gemacht. - Kann man aber sagen, jede existierende Person habe mit analytischer Notwendigkeit Masse?
\end{abstract}


moderner Deutung zustimmt, sondern vielmehr in einer anderen, alten. (Sonst müßte man ihm einen begriffsanalytischen Fehler unterstellen.) Für ihn ist eine Person eine Seele (eine res cogitans also etwas, womit eine Seele korreliert ist, d.h. Seele oder etwas Beseeltes). ${ }^{19}$ $\mathrm{Daß}$ Descartes essentiell eine Person in diesem Sinne ist, ist verträglich damit, daß er existieren kann ("Können" im weitesten Sinne), ohne eine res extensa zu sein; dagegen, daß Descartes essentiell eine Person im modernen Sinn ist, ist unverträglich damit, daß er existieren kann, ohne eine res extensa zu sein.

Ist die moderne Deutung die richtige, und wenn ja, in welchem Sinne ist sie die richtige? Dies freilich sind Fragen, die hier nicht beantwortet werden können. Es liegt jedenfalls nicht auf der Hand, in welchem Sinne die Deutung Descartes' die falsche ist.

19. F. Sommers schreibt in seinem Aufsatz "Dualism in Descartes: The Logical Ground" (in Descartes: Critical and Interpretive Essays, hrsg. von M. Hooker, Baltimore 1978) auf S. 223: "According to Descartes, a person is an ontologically composite entity in the sense of being composed of entities of different types." Sommers nennt keine Textstelle zum Beleg dieser Behauptung. Sie ist aber unrichtig oder paßt zumindest nicht in den Kontext der Meditationes. Descartes sagt nicht "ich (d.h. eine Person) bin aus Körper und Seele zusammengesetzt", sondern "ich (d.h. eine Person) bin mit einem Körper verbunden", woraus hervorgeht, daß er unter einer Person etwas in sich Unkörperliches versteht.

\section{Literatur:}

Zur Modallogik und zur epistemisch-doxastischen Logik, die in diesem Aufsatz zur Anwendung kommt, siehe: F. v. Kutschera, Einführung in die intensionale Semantik, Berlin 1976, insbesondere Kapitel 2 und 4.

Im Text erwähnte und diskutierte Literatur:

Alanen, L.: Studies in Cartesian Epistemology and Philosophy of Mind, Helsinki 1982;

Beckermann, A.: Descartes' metaphysischer Beweis für den Dualismus, Freiburg/München 1986;

Gueroult, M.: Descartes selon Pordre des raisons, II, Paris 1968; 
Hooker, M.: “Descartes's Denial of Mind-Body Identity" in M. Hooker (Hrsg.), Descartes: Critical and Interpretive Essays, Baltimore/London 1978, S.171-S.185;

Kenny, A.: The God of the Philosophers, Oxford 1979;

Kripke, S.: "Identity and Necessity" in M.K. Munitz (Hrsg.), Identity and Individuation, New York 1971, S.135-164;

Kutschera, F. v.: Grundfragen der Erkenntnistheorie, Berlin 1982;

Ryle, G.: The Concept of Mind, London 1949;

Sommers, F.: "Dualism in Descartes: The Logical Ground" in M. Hooker (Hrsg.), Descartes: Critical and Interpretive Essays, Baltimore/London 1978, S.223-S.233;

Wilson, M.D.: Descartes, London 1978. 